\title{
Human Extracellular-Matrix Functionalization of 3D hiPSC-Based Cardiac Tissues Improves Cardiomyocyte Maturation
}

\author{
Henrique V. Almeida," Miguel F. Tenreiro," Ana F. Louro, Bernardo Abecasis, Deolinda Santinha, \\ Tomás Calmeiro, Elvira Fortunato, Lino Ferreira, Paula M. Alves, and Margarida Serra*
}

Cite This: ACS Appl. Bio Mater. 2021, 4, 1888-1899

Read Online

\section{ACCESS | Lill Metrics \& More | 回 Article Recommendations ｜（） Supporting Information}

ABSTRACT: Human induced pluripotent stem cells (hiPSC) possess significant therapeutic potential due to their high self-renewal capability and potential to differentiate into specialized cells such as cardiomyocytes. However, generated hiPSCderived cardiomyocytes (hiPSC-CM) are still immature, with phenotypic and functional features resembling the fetal rather than their adult counterparts, which limits their application in cell-based therapies, in vitro cardiac disease modeling, and drug cardiotoxicity screening. Recent discoveries have demonstrated the potential of the extracellular matrix (ECM) as a critical regulator in development, homeostasis, and injury of the cardiac microenvironment. Within this context, this work aimed to assess the impact of human cardiac ECM in the phenotype and maturation features of hiPSC-CM. Human ECM was isolated from myocardium tissue through a physical decellularization approach. The cardiac tissue decellularization process reduced DNA content significantly while maintaining ECM composition in terms of sulfated

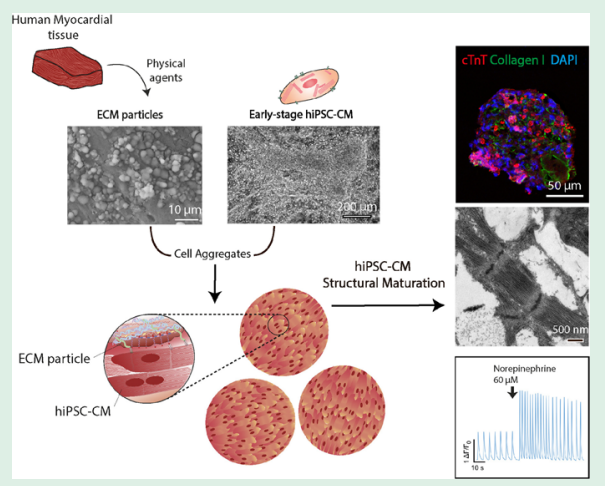
glycosaminoglycans (s-GAG) and collagen content. These ECM particles were successfully incorporated in three-dimensional (3D) hiPSC-CM aggregates (CM+ECM) with no impact on viability and metabolic activity throughout 20 days in 3D culture conditions. Also, CM+ECM aggregates displayed organized and longer sarcomeres, with improved calcium handling when compared to hiPSCCM aggregates. This study shows that human cardiac ECM functionalization of hiPSC-based cardiac tissues improves cardiomyocyte maturation. The knowledge generated herein provides essential insights to streamline the application of ECM in the development of hiPSC-based therapies targeting cardiac diseases.

KEYWORDS: extracellular matrix, hiPSC-CM, 3D culture, cardiac tissue decellularization, cardiomyocyte maturation

\section{INTRODUCTION}

The adult human heart has a negligible capacity to regenerate, with a cardiomyocyte renewal rate estimated at $1 \%$ each year, which declines with age. ${ }^{1}$ Even if adult cardiomyocytes isolated from cardiac biopsies were an unlimited cell source for cardiac research, dedifferentiation and loss of viability in vitro soon after isolation discourage their use. ${ }^{2}$ Although adult cardiac tissue may be kept under electromechanical stimuli, noticeable alterations at the structural, mechanical, and gene expression levels are still detectable. ${ }^{3}$ Therefore, cardiac research uses other platforms, such as animal models and ion-channel overexpressing cell lines, even though they present overwhelming physiological differences relative to human cardiac tissue. Because of their high self-renewal ability and capacity to differentiate into any cell type of the human body, including cardiac cell lineages, human induced pluripotent stem cells (hiPSC) have been establishing a new paradigm in cardiac research. Their capability to generate high numbers of patientspecific and functional cardiomyocytes makes them a powerful cell source to be used not only in personalized cell therapy ${ }^{4}$ but also in drug screening ${ }^{5}$ and disease modeling. ${ }^{6}$ In the past decade, much effort has been made to develop improved protocols for the generation of hiPSC-CM in high yields and purity with electrophysiological and contractile properties.

Despite their promising features, generated hiPSC-CM still maintain an immature state following differentiation. ${ }^{8}$ The fetal-like phenotype, structure, and functionality of these cells dramatically limit their translational use, and thus, several strategies are under evaluation to allow these cells to mature and resemble those found in an adult human heart. Within this context, the impact of critical environmental and biological factors, including (i) mechanical ${ }^{9,10}$ and electrical stimuli, ${ }^{10-12}$ (ii) co-culture strategies with non-myocytes cells, ${ }^{13,14}$ (iii) metabolic modulation, ${ }^{15,16}$ (iv) hormonal treatment, ${ }^{17,18}$ and (v) 3D culturing approaches, ${ }^{19-21}$ on hiPSC-CM maturation have been reported. The most advanced strategies reported to date have been successful in even emulating certain

Received: November 27, 2020

Accepted: January 20, 2021

Published: January 29, 2021 
cardiomyocyte features that only develop after birth such as a vast network of T-tubules that ensure calcium homeostasis and a rapid excitation-contraction coupling (ECC), adequate inotropic response, reliance on oxidative metabolism through $\beta$-oxidation, a positive force-frequency relationship (FFR), and greater contractile force to ensure pump function. ${ }^{10,12,20}$ To obtain such mature features, hiPSC-CM are typically assembled into engineered heart tissues (EHT) or complex 3D scaffolding materials, which compromises their highthroughput performance. Furthermore, growing these tissues requires a suitable biomaterial, most commonly single-protein extracellular matrix (ECM)-derived products derived from animal tissue (e.g., rat-tail collagen), proteins that have little presence in the fetal or adult heart (e.g., fibrin), or even more complex matrices subjected to batch-to-batch variability (e.g., Matrigel). Despite their adequate biological activity, these biomaterials are far from providing the same biochemical and biophysical cues present in the native cardiac ECM. Nevertheless, the potential of engineered functional biomaterials that mimic the ECM has been widely explored in the literature. When combined with stem cells or their derivatives, these specialized materials can enable robust biological mimicry for efficient tissue engineering. ${ }^{22-24}$

The cardiac ECM is a complex fibrillar protein network of glycosaminoglycans (GAG), and proteoglycans, arranged in a precise $3 \mathrm{D}$ framework that accommodates cardiomyocytes, fibroblasts, endothelial cells, and other non-myocyte cells in the heart, besides providing an ideal milieu for multiple cellreceptor and growth factors. ${ }^{25}$ Both in vivo and in vitro studies suggest that the cardiac ECM could have a significant role in cell/tissue differentiation, development, and maturation. For instance, the ECM protein agrin triggers mild cardiomyocyte differentiation and proliferation in mice, besides being fundamental in regeneration at neonatal stages. ${ }^{26}$ In addition, the effect of laminin is well documented regarding development and regeneration, ${ }^{27}$ and particularly laminin was identified as a potent enhancer of cardiomyocyte maturation. ${ }^{28}$ Decellularized mouse heart ECM further aids the differentiation of embryonic stem cells toward cardiomyocytes. ${ }^{29}$ Moreover, the age (fetal versus adult) and geometry (2D versus $3 \mathrm{D}$ ) of decellularized porcine heart ECM modulate the mechanical properties as well as fiber architecture and were shown to influence hiPSC-CM maturation at gene and protein expression levels. $^{30}$ Although human ECM isolated from perfusion-decellularized hearts could sustain contracting hiPSC-CM into bundle-like tissues, ${ }^{31}$ the authors did not evaluate its impact on the cells' maturation profile.

Herein, we evaluated the effect of ECM of adult human myocardium tissue isolated from healthy donors, in the phenotype and maturation features of hiPSC-CM aggregated into a 3D microtissue. Human myocardial tissue was physically processed, without detergents or enzymes, into homogeneous ECM particles that were characterized at the physical and biochemical level and, for the first time, incorporated in the 3D hiPSC-derived cardiac aggregate model, developed previously by our group. ${ }^{19}$ These ECM functionalized hiPSC-CM aggregates were cultured for 20 days, and their phenotype, structure, and functionality were evaluated and compared with hiPSC-CM aggregates. We believe that our approach is innovative by using cryomilled human ECM to mature hiPSC-CM in 3D aggregates. Furthermore, this approach presents several advantages compared with hydrogels with solubilized ECM, including the maintenance of the cardiac tissue's micromechanical and compositional properties.

\section{MATERIALS AND METHODS}

2.1. Human Cardiac Tissue Processing. Human heart tissue biopsies were provided by Coimbra Hospital University Centre (CHUC, Coimbra, Portugal) following the regulations of the tissue bank and the approval of the Ethics Committee of Faculty of Medicine of the University of Coimbra (CE-022/2017). Left ventricular myocardial tissues were harvested, by the hospital unit, from the heart of donors $(n=3)$ with no pathology associated, that were not suitable for transplantation, after specific consent was obtained; donor ages were 67 (female), 67 (female), and 69 (male). Shortly after harvest, myocardial tissue samples were stored at -80 ${ }^{\circ} \mathrm{C}$. In this work, a physical-based protocol was applied for decellularization of the myocardial tissue. Briefly, myocardial tissue samples were thawed, pooled, and sectioned into small pieces using scalpels, in aseptic conditions. The tissue pieces were then further fragmented using a manual cryomilling method, as described further. The tissue pieces were rinsed in Dulbecco's phosphate-buffered saline (DPBS, Thermo Fisher Scientific), followed by a freezing step in liquid nitrogen. The samples were milled aseptically using a mortar and pestle to produce a fine powder. This cryomilling was repeated three times, and the resulting material was washed and recovered with DPBS and stored at $-80^{\circ} \mathrm{C}$. ECM particles were then freeze-dried (freeze-drier Labconco Triad) overnight and stored at $-80{ }^{\circ} \mathrm{C}$.

2.2. Characterization of Human Cardiac ECM. 2.2.1. Histology: ECM Particles. The ECM particles were fixed in $4 \%(\mathrm{w} / \mathrm{v})$ buffered paraformaldehyde (PFA, Sigma-Aldrich) for $18 \mathrm{~h}$ at $4{ }^{\circ} \mathrm{C}$, washed twice in DPBS, pelleted, and embedded in $2 \%(\mathrm{w} / \mathrm{v})$ high melting temperature agarose (Lonza). Samples were dehydrated at room temperature (RT, 20-22 ${ }^{\circ} \mathrm{C}$ ), paraffin-embedded, and sectioned (3 $\mu \mathrm{m}$ thickness) on a rotary microtome (RM 2135, Leica). Sections were subjected to standardized deparaffinization procedure and then stained with hematoxylin and eosin (H\&E, Sigma-Aldrich) and Masson's Trichrome with Aniline Blue (MT, IHC World), according to the manufacturers' instructions. Histological images were digitalized in a NanoZoomer SQ whole slide scanner (Hamamatsu Photonics) and analyzed with NDP.view open-source software (NDP.view v.2.7.43, Hamamatsu Photonics).

2.2.2. Biochemical Analysis: dsDNA and ECM Components Quantification. Double-stranded DNA (dsDNA) and key ECM components were quantified in decellularized ECM. Decellularized samples were digested in $500 \mu \mathrm{L}$ of papain solution $(125 \mu \mathrm{g} / \mathrm{mL}$ in $0.1 \mathrm{M}$ sodium acetate, $5 \mathrm{mM}$ cysteine- $\mathrm{HCl}, 0.05 \mathrm{M}$ EDTA, $\mathrm{pH}$ 6.0, all from Sigma-Aldrich) for $20 \mathrm{~h}$ at $60{ }^{\circ} \mathrm{C}$ under agitation. Native tissue samples with similar wet weight were also digested. The dsDNA content was determined using the Quanti-iTPicoGreen assay (Thermo Fisher Scientific) according to the manufacturer's instructions. The fluorescent intensity was measured in the microplate reader Infinite200 PRO (NanoQuant, Tecan Trading AG). The sulfated glycosaminoglycans (s-GAG) content was quantified using the 1,9-dimethyl methylene blue assay (Glycosaminoglycan-GAG Assay Blyscan, Biocolor), and collagen content (soluble, insoluble, and total) was determined via a commercial kit (Sircol Collagen Assay, Biocolor). Sample absorbance was measured in the plate reader Infinite200 PRO. Data were expressed as the measured component mass normalized for tissue sample wet weight.

2.2.3. Scanning Electron Microscopy and Energy-Dispersive $X$ ray Spectroscopy of ECM Particles. ECM particles were fixed in $4 \%$ $(\mathrm{w} / \mathrm{v})$ PFA $\left(4^{\circ} \mathrm{C}\right.$ for $\left.18 \mathrm{~h}\right)$, followed by three washes with DPBS for $10 \mathrm{~min}$ at RT. Samples were dried in adhesive carbon substrates (12 $\mathrm{mm}$, Agar Scientific), sputter-coated with a $15 \mathrm{~nm} \mathrm{Au} / \mathrm{Pd}$ film, and visualized in a tabletop scanning electron microscope with energydispersive X-ray spectroscopy capability (SEM, Hitachi TM3030Plus; EDS, Bruker QUANTAX) operated in backscattered electron imaging mode at an accelerating voltage of $15 \mathrm{keV}$. SEM images of ECM particles were analyzed in ImageJ open-source software (Rasband, W.S., ImageJ, U.S. National Institutes of Health) to estimate particle mean Feret diameter. 
2.2.4. Atomic Force Microscopy of ECM Particles. Hydrated ECM particles were dried in a vacuum chamber and immobilized with double side tape. Atomic force microscopy (AFM) analysis was done with an Asylum Research MFP-3D Standalone system operated in AC (alternate contact) mode in air, using commercially available silicon probes (Olympus AC160TS; $k=26 \mathrm{~N} / \mathrm{m} ; f_{0}=300 \mathrm{kHz}$ ). The resulting topographs were low-order plane fitted before being exported as images using Gwyddion software.

2.3. hiPSC Culture. 2.3.1. Expansion and Differentiation of hiPSC into Cardiomyocytes. The hiPSC line DF19-9-11T.H (WiCell) was used in this study, and cells were propagated and differentiated into cardiomyocytes according to the protocol developed by our group. ${ }^{19}$ The hiPSC were propagated in Matrigelcoated (Corning) plates as previously described. ${ }^{19,32}$ At day 8 of differentiation, cells, designated as early stage cardiomyocytes, were detached with TrypLE Select (Thermo Fisher Scientific) as reported elsewhere. ${ }^{19,32}$

2.3.2. Generation of ECM Functionalized hiPSC-CM Aggregates. The ECM particles were immersed in $70 \%$ (v/v) ethanol for $30 \mathrm{~min}$ at RT and treated with ultraviolet light $(15 \mathrm{~min})$. After being sterilized, ECM particles were rinsed with DPBS and air-dried aseptically. Cells harvested at day 8 of differentiation were resuspended in RPMI medium supplemented with B27 with insulin (RPMI/B27 plus insulin, Thermo Fisher Scientific), containing 1\% (w/v) ECM particles (corresponding to $3 \mathrm{ng}$ of ECM per cell). The cell aggregates functionalized with ECM particles (hereafter designated as $\mathrm{CM}+\mathrm{ECM}$ aggregates) were obtained by forced aggregation in AggreWell400Ex (Stem Cell Technologies), and cultured in orbital suspension cultures (28 days), as previously described by our group. ${ }^{19}$ In parallel, we performed forced aggregation of cells without ECM as control (hereafter designated as $\mathrm{CM}$ aggregates).

2.4. Characterization of hiPSC-CM Aggregates. 2.4.1. Cell Viability, Beating Rate, and Metabolic Activity. The qualitative cell viability was assessed by fluorescein diacetate (FDA Sigma-Aldrich), and cell membrane integrity with the DNA-binding dye propidium iodide (PI, Sigma-Aldrich), following the manufacturer instructions, and the aggregates were visualized in a fluorescent microscope (DMI600, Leica). The cells capable of accumulating the product of FDA metabolization were stained fluorescent green and were considered to be alive, while cells stained fluorescent red with PI were considered dead. The average Feret diameter of cell aggregates was estimated in ImageJ by analyzing several frames collected during live imaging (at least 250 cell aggregates were analyzed per condition). Also, cells were counted using Trypan Blue (Thermo Fischer Scientific), as previously described. ${ }^{33}$

The beating rate was estimated by visualizing live-cell aggregates under an inverted microscope (Olympus CKX31, Olympus) on a heat plate (KL $1500 \mathrm{LCD}$, Leica) set at $37^{\circ} \mathrm{C}$; at least $n=3$ cell aggregates were analyzed per time point and condition.

The aggregate metabolic activity was quantified, as previously described by our group, ${ }^{34}$ with PrestoBlue (Life Technologies), following the manufacturer's instructions.

2.4.2. Histology and Polarized Light Microscopy of hiPSC-CM Aggregates. The histological sectioning of hiPSC-CM aggregates and staining was done as described earlier (section 2.2.1). Samples were either stained with H\&E, MT, or Picro Sirius Red (PSR, Polysciences), according to the manufacturer's guidelines. PSRstained samples were visualized under a microscope with standard bright field and polarization (Olympus BX41TF-P Polarized Light Microscope, Olympus).

2.4.3. Scanning Electron Microscopy Analyzes of hiPSC-CM Aggregates. The cell aggregate samples were collected and fixed with $4 \%(\mathrm{w} / \mathrm{v})$ PFA $\left(4{ }^{\circ} \mathrm{C}\right.$ for $\left.18 \mathrm{~h}\right)$ and rinsed with DPBS (RT for 10 $\mathrm{min})$. Samples were dried in $12 \mathrm{~mm}$ adhesive carbon tape, sputtercoated with a $15 \mathrm{~nm} \mathrm{Au} / \mathrm{Pd}$ film, and visualized in a ZEISS Auriga Crossbeam SEM $(5 \mathrm{keV})$.

2.4.4. Biochemical Analysis of hiPSC-CM Aggregates. Biochemical analysis was carried out as indicated earlier (section 2.2.2). For cell aggregates, data were expressed as the measured ECM component mass normalized for measured dsDNA mass.

2.4.5. Immunofluorescence Microscopy of hiPSC-CM Aggregates. The cell aggregates were harvested at day 28 and fixed in $4 \%$ $(\mathrm{w} / \mathrm{v})$ PFA with $4 \%(\mathrm{w} / \mathrm{v})$ sucrose in DPBS (RT for $20 \mathrm{~min})$. The samples were cryosectioned and the immunofluorescence performed as previously described by our group. ${ }^{34}$ The primary antibodies used were cardiac troponin $\mathrm{T}(\mathrm{cTnT})$ (Thermo Fisher Scientific, diluted 1:200), sarcomeric $\alpha$-actinin (Sigma-Aldrich, diluted 1:200), collagen I (abcam, diluted 1:100), $\alpha$-smooth muscle actin ( $\alpha$ SMA; Agilent Technologies/DAKO, diluted 1:100), and vimentin (abcam, diluted $1: 100)$. The secondary antibodies used were goat antirabbit IgG Alexa Fluor 486 and goat antimouse Alexa Fluor 594 (all from Thermo Fisher Scientific, diluted 1:500). The samples were visualized in a confocal microscope (SP5 Live, Leica).

2.4.6. Flow Cytometry. The hiPSC-CM aggregates were collected at day 28 and dissociated with TrypLE $\left(37^{\circ} \mathrm{C}\right.$ for $5 \mathrm{~min}$ ) for flow cytometry analysis as previously described by our group. ${ }^{34}$ The following conjugated antibodies were used: $\operatorname{SIRP} \alpha / \beta$ (CD172a/b-PE, BioLegend, diluted 1:20 in DPBS) and isotype control IgG2, k-PE (BD Biosciences, diluted 1:5 in DPBS). Stained cell suspensions were analyzed in a BD FACSCalibur cytometer (BD Biosciences), and the analysis was performed with Flowjo software (TreeStar).

2.4.7. Gene Expression Analysis. Total RNA of aggregates collected at days 11,20 , and 28 of culture was isolated, and the gene expression analysis was performed by real-time quantitative polymerase chain reaction (RT-qPCR) using TaqMan Gene Expression Assays (MYH7, Hs01110632 m1; MYH6, Hs01101425 m1; MYL2, Hs00166405 m1; MYL7, Hs00221909 m1; TNNI3, Hs00165957 m1; GAPDH, Hs99999905 m1; all from Thermo Fisher Scientific), as previously described by our group. ${ }^{34}$ The data were analyzed with $\log _{2}$-fold change normalized to day 11 of each experimental condition and to GAPDH gene expression.

2.4.8. Transmission Electron Microscopy of hiPSC-CM Aggregates. The samples were fixed in $2.5 \%(\mathrm{v} / \mathrm{v})$ glutaraldehyde in DPBS (RT for $20 \mathrm{~min}$ ). The sample preparation and analysis were performed as previously described. ${ }^{34}$ The transmission electron microscopy (TEM) image analysis was done using ImageJ. Z-lines corresponded to electron-dense regions, and the repeating units between Z-lines were identified as the sarcomeres. The sarcomere length was determined as the distance between consecutive parallel Zlines and Z-line length as the length of the electron-dense regions. Sarcomere alignment was estimated based on the magnitude of angle dispersion. Briefly, for frames containing several sarcomeres, the angles of sarcomere edges were measured, the standard error of the mean of angle measurements was computed, and data were presented as the mean of those values. A low angle of dispersion is indicative of a higher alignment degree.

2.4.9. Calcium Imaging. The calcium handling was evaluated with the Fluo-4 Direct Calcium Assay Kit (Thermo Fisher Scientific), as previously described. ${ }^{13,34}$ The results were analyzed using a custom MATLAB script (MATLAB R2017b, Mathworks). The data were normalized to baseline fluorescence $\left(\Delta F / F_{0}\right)$, and each transient calcium peak was analyzed for the following parameters: amplitude, time to $80 \%$ decay, time to peak, maximal upstroke velocity, maximal decay velocity, and cycle length, following the methodology described before. ${ }^{13}$ In brief, calcium transient amplitude corresponds to the maximal normalized fluorescent intensity; $80 \%$ decay time corresponds to the time interval between maximal normalized fluorescent intensity and $20 \%$ normalized fluorescent intensity during repolarization; time to peak was determined as the time interval between minimum and maximal normalized fluorescent intensity during depolarization; maximal upstroke and maximal decay velocities correspond to the maximum magnitude of the tangent slopes to a calcium transient during depolarization and repolarization, respectively; cycle length was computed as the time interval a calcium transient lasts.

2.5. Statistical Analysis. Data are shown as mean \pm standard deviation of independent measurements or assays (minimum of $n=$ 3), and statistical analysis was performed using GraphPad Prism 7 

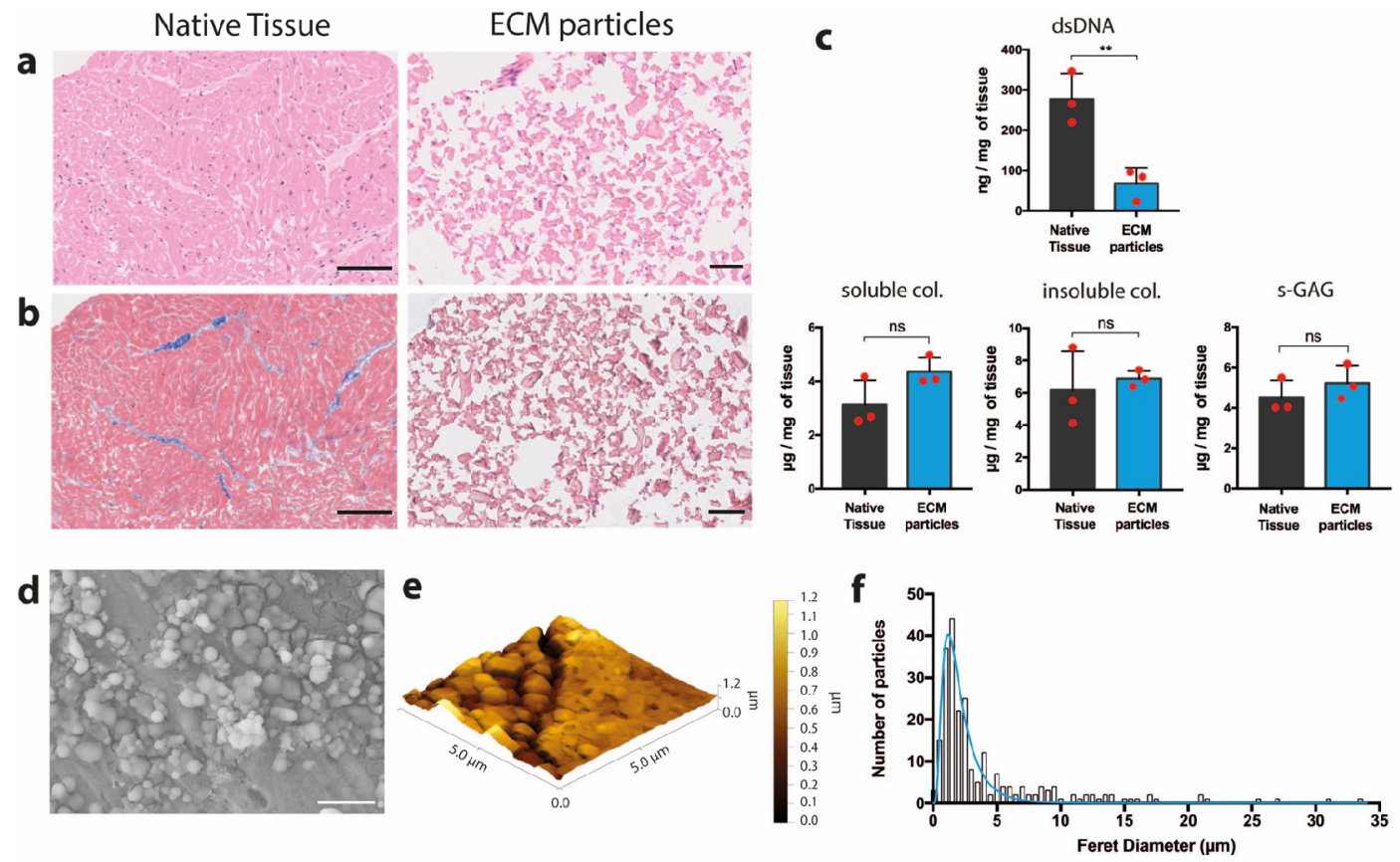

Figure 1. Biochemical and structural characterization of physical-based decellularized human myocardial tissue. (a) Hematoxylin and Eosin (H\&E) and (b) Masson's Trichrome (MT) staining for human myocardial native tissue and ECM particles. ECM particles were obtained by pooling samples from the three tissue donors. Scale bars: $100 \mu \mathrm{m}$ (native tissue, left panels) and $50 \mu \mathrm{m}$ (ECM particles, right panels). (c) Double-stranded DNA (dsDNA), sulfated glycosaminoglycans (s-GAG), soluble collagen, and insoluble collagen content on native tissue and ECM particles. The average of the three myocardial tissue donors was used as a representative value for the component in the myocardium and normalized to tissue wet weight $(\mathrm{mg})$. Data are presented as mean \pm standard deviation $(\mathrm{SD})$ of three biological replicates (red dots corresponding to each tissue donor). $*_{*}^{*} p<0.01$; ns, not significant. (d) Scanning electron microscopy (SEM) of ECM particles. Scale bar: $10 \mu \mathrm{m}$. (e) Atomic force microscopy (AFM) 3D scanning image of ECM particles. (f) Histogram of Feret diameter distribution for ECM particles ( $n=235$ measurements). The blue line is a log-normal curve fitting to the data.

(GraphPad Software). The differences between experimental groups were analyzed by Student's $t$ test or by one-way ANOVA. Post hoc pairwise analysis was done using Tukey's HSD test; $p$-values $<0.05$ were considered significant for all statistical tests.

\section{RESULTS}

3.1. Processing and Characterization of Physically Decellularized Human Myocardial Tissue. Adult myocardial tissues derived from three healthy donors were assessed histologically, showing typical myocardial architecture (i.e., muscle bundles) with the staining of traditional cellular and ECM components such as nuclei and cytoplasm (HE), and collagen (MT) (Figure 1a,b, left panels). Physical agents, including temperature, ${ }^{35}$ force, ${ }^{36}$ and pressure, ${ }^{37,38}$ can be used to effectively decellularize biological tissue. After thawing, myocardial tissue pieces were snap-frozen in liquid nitrogen, milled at room temperature, and freeze-dried overnight. Cellular and matrisomal tissue organization were disrupted throughout this process, creating tissue particles, designated as ECM particles. By histology, loss of tissue morphological architecture becomes evident (Figure 1a,b, right panels). The efficiency of decellularization was evaluated through dsDNA quantification assay. Native myocardial tissue contained 277.10 $\pm 37.07 \mathrm{ng} / \mathrm{mg}$ wet tissue, and decellularized tissue had 67.87 $\pm 22.77 \mathrm{ng} / \mathrm{mg}$ wet tissue of residual dsDNA (Figure 1c), corresponding to a $75.5 \%$ reduction. $\mathrm{H} \& \mathrm{E}$ staining of ECM particles showed little nuclei staining and cell debris after decellularization (Figure 1a,b, right panels), supporting dsDNA reduction. The impact of decellularization was also evaluated concerning ECM composition by quantifying soluble and insoluble collagen (hydroxyproline) and s-GAG before and after physical treatment. Decellularization did not cause a significant loss of soluble collagen $(4.36 \pm 0.31 \mu \mathrm{g} / \mathrm{mg}$ wet tissue), insoluble collagen $(6.88 \pm 0.29 \mu \mathrm{g} / \mathrm{mg}$ wet tissue $)$, and $\mathrm{s}$-GAG (5.23 $\pm 0.50 \mu \mathrm{g} / \mathrm{mg}$ wet tissue) (Figure 1c), even though their organization within the ECM is not preserved (Figure 1a,b, right panels).

SEM-EDS and AFM analyses of ECM particles suggested that the decellularization protocol generated micrometric sized particles, with a spherical-like shape (Figures $1 \mathrm{~d}$ and S1) and irregular surface topography (Figure 1e). ECM particles showed a mean Feret diameter of $4.27 \mu \mathrm{m}$ and low polydispersity (Figure 1f), demonstrating the robustness of the protocol in generating uniformly sized decellularized particles. These ECM particles were used in the functionalization of 3D hiPSC-CM aggregates, as described further.

3.2. Establishment of a Functionalized Cardiac Aggregate Tissue Model. Scaffold-free 3D cardiac tissues, such as hiPSC-CM aggregates, are powerful tools for high throughput analysis, even though they do not yet fully recapitulate the cardiac microenvironment. To improve their physiological relevance, we incorporated human ECM components in these microtissue models, namely micrometric ECM particles isolated and processed from healthy adult cardiac tissue. Briefly, early stage hiPSC-CM were harvested from $2 \mathrm{D}$ contracting monolayers cultures at day 8 of differentiation, as previously described by our group, ${ }^{19}$ and aggregated using AgreeWell plates in the presence and absence of $1 \%(\mathrm{w} / \mathrm{v}) \mathrm{ECM}$ particles (corresponding to $3 \mathrm{ng} \mathrm{ECM} /$ cell), hereafter designated as $\mathrm{CM}+\mathrm{ECM}$ and $\mathrm{CM}$ groups, 


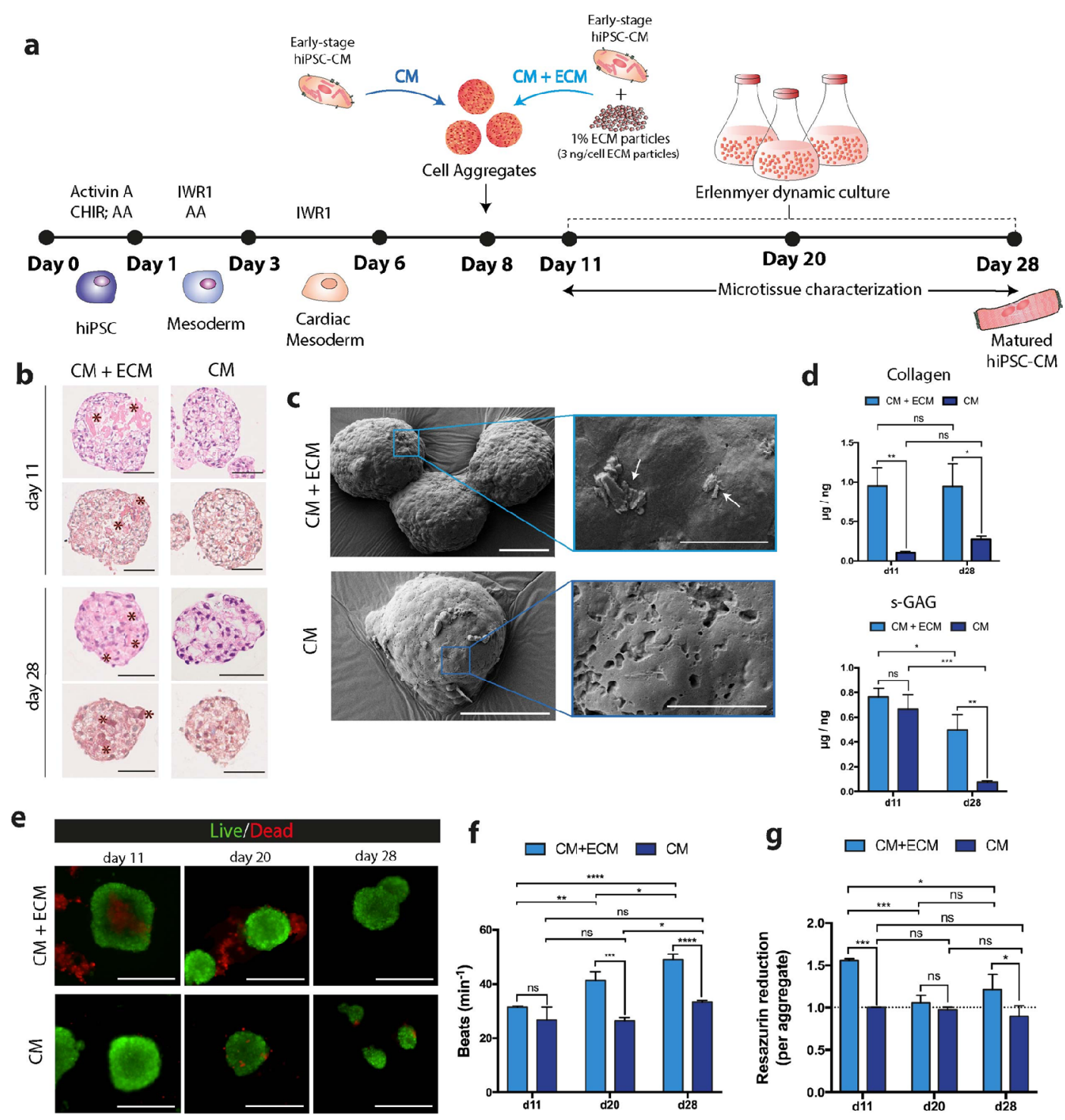

Figure 2. ECM particles can be incorporated in a 3D cardiac aggregate model without compromising cell viability. (a) Experimental design: early stage human induced pluripotent stem cell-derived cardiomyocytes (hiPSC-CM) were aggregated without (CM) or in combination with $1 \%(\mathrm{w} / \mathrm{v}$ ) (i.e., $3 \mathrm{ng} /$ cell) ECM particles obtained from a physical-based decellularization method (CM+ECM). Aggregates were kept in Erlenmyer dynamic culture for 17 days. (b) Hematoxylin and Eosin and Masson's Trichrome staining for CM+ECM and CM aggregates at the beginning and end of the culture period. (*) denotes ECM particles within CM+ECM aggregates. Scale bars: $50 \mu \mathrm{m}$. (c) Scanning electron microscopy (SEM) of CM $+\mathrm{ECM}$ and $\mathrm{CM}$ aggregates at day 28 showing zoomed in sections detailing aggregate surface topography. White arrows point to ECM particles present in the surface of a representative $\mathrm{CM}+\mathrm{ECM}$ aggregate. Scale bars: $50 \mu \mathrm{m}$ (left panels) and $5 \mu \mathrm{m}$ (right panels). (d) Biochemical analysis of total collagen (hydroxyproline) and sulfated glycosaminoglycan (s-GAG) content of CM+ECM and CM aggregates $(n=3$ per time-point per condition). Normalized to double stranded DNA weight. (e) Viability analysis of the CM+ECM and CM aggregates throughout culture time by fluorescence microscopy, stained with fluorescein diacetate (FDA-live cells, green) and propidium iodide (PI-dead cells, red). Scale bars: $200 \mu \mathrm{m}$. (f) Beating rate of CM+ECM and CM aggregates through culture time ( $n=3$ per time-point per condition). (g) Metabolic activity of CM+ECM and $\mathrm{CM}$ aggregates throughout culture time (Presto-Blue assay), normalized by aggregate and to CM condition at day 11 ( $n=3$ per time point per condition). Throughout, data are presented as mean $\pm \mathrm{SD} * p<0.05, * * p<0.01, * * * p<0.001, * * * * p<0.0001$, ns, not significant, determined by one-way ANOVA followed by Tukey's HSD test. CHIR, CHIR99021; AA, Ascorbic acid.

respectively (Figure 2a). Three days after forced aggregation, cell aggregates were transferred to Erlenmeyer flasks and cultured in agitated conditions for additional 17 days (total culture time: 28 days) (Figure $2 \mathrm{a}$ ).

Histological sections of cell aggregates confirmed the incorporation of the ECM particles within $\mathrm{CM}+\mathrm{ECM}$ aggregates and their presence from the beginning to the end of the 3D culture period (Figure 2b). CM+ECM aggregates appear to be more compact, possibly owing to early lumen filling by ECM particles. SEM analysis also revealed the presence of ECM particles on the surface of $\mathrm{CM}+\mathrm{ECM}$ aggregates (Figure 2c). It is important to mention that these ECM motifs are not present in the control group with CMonly.

Biochemical analysis of aggregate content revealed higher collagen and s-GAG concentration in $\mathrm{CM}+\mathrm{ECM}$ aggregates throughout culture time (Figure $2 \mathrm{~d}$ ). Collagen levels at day 11 in $\mathrm{CM}+\mathrm{ECM}$ aggregates $(0.95 \pm 0.23 \mu \mathrm{g} / \mathrm{ng}$ of dsDNA) were significantly higher than in CM aggregates $(0.10 \pm 0.02 \mu \mathrm{g} / \mathrm{ng}$ of dsDNA), indicating incorporation of ECM particles within 
a

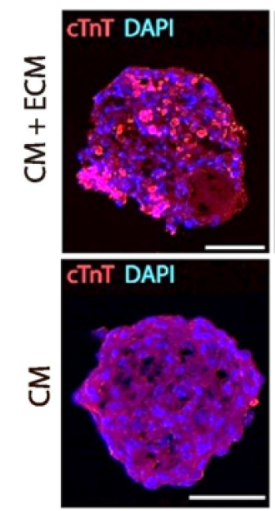

d
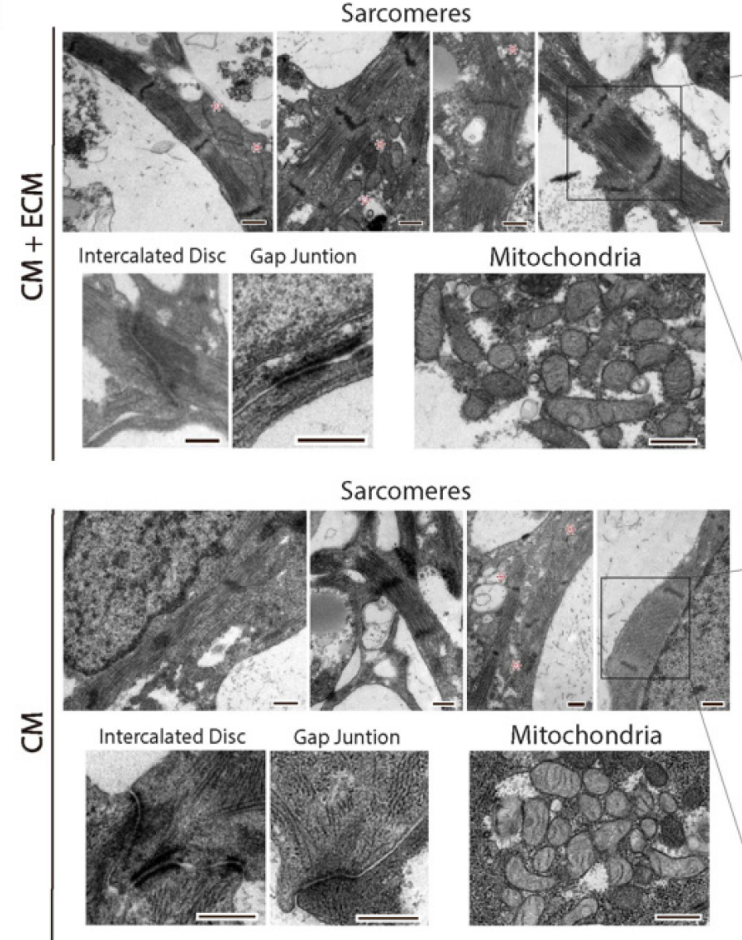

b
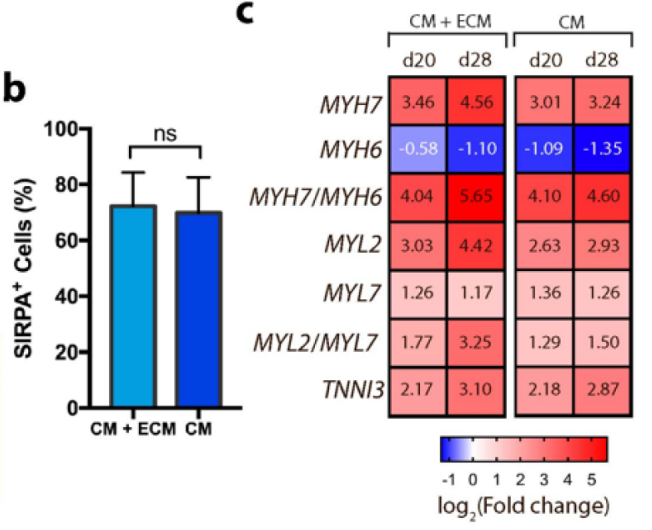

e
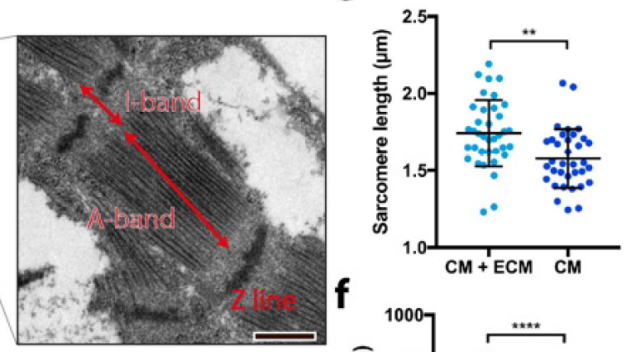

f

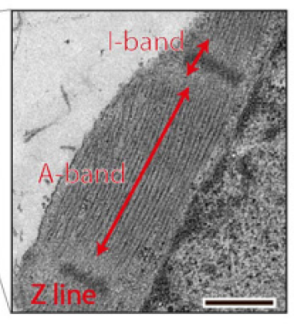

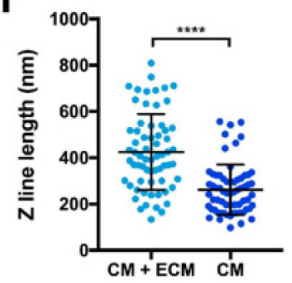

g

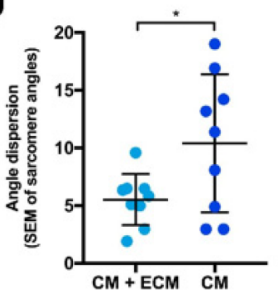

Figure 3. Evidence of phenotypic, structural, and ultrastructural maturation in $\mathrm{CM}+\mathrm{ECM}$ aggregates. (a) Immunofluorescence microscopy of CM $+\mathrm{ECM}$ and CM aggregates cryosections at day 28. The cells were stained for sarcomeric structural proteins (cardiac troponin T (cTnT) and $\alpha$ actinin, red) and DAPI (blue). Zoomed-in sections show evidence of apparent typical cardiomyocyte striation. Scale bar: $50 \mu \mathrm{m}$; $10 \mu \mathrm{m}$ (zoom). (b) Percentage of SIRP $\alpha / \beta^{+}$cells dissociated from CM+ECM and CM aggregates at day 28 ( $n>2$ per condition). (c) Gene expression data of CM $+\mathrm{ECM}$ and $\mathrm{CM}$ aggregates throughout culture time for sarcomeric isoforms of mature and immature ventricular phenotype. The gene expression was normalized to day 11 to each condition, and the mean fold change is presented ( $n=3$ per time point per condition). (d) Transmission electron microscopy (TEM) images of CM+ECM and CM aggregates at day 28 showing aligned myofibrils, composed by sarcomeres with organized $\mathrm{Z}$ lines, A-bands, and I-bands; cell junction (intercalated discs and gap junctions) between adjacent cardiomyocytes; abundant mitochondria. (*) denotes mitochondria near sarcomeres. Scale bar: $500 \mathrm{~nm}$. (e) Mean sarcomere length of CM+ECM and CM aggregates $(n=35$ measurements per condition). (f) Mean $\mathrm{Z}$ line length of $\mathrm{CM}+\mathrm{ECM}$ and $\mathrm{CM}$ aggregates $(n=65$ measurements per condition). (g) Sarcomere alignment was determined by myofibril angle dispersion of CM+ECM and CM aggregates $(n=9$ measurements per condition). Throughout, data are presented as mean \pm standard deviation $(\mathrm{SD}) . *_{p}<0.05, *^{*} p<0.01, * * * p<0.0001$, ns, not significant, determined by Student's $t$ test.

aggregates and provided an initial collagen enriched microenvironment (Figure 2d, upper panel). By day 28, no significant changes in collagen concentration from initial values were found in CM+ECM $(0.94 \pm 0.29 \mu \mathrm{g} / \mathrm{ng}$ of dsDNA $)$ and CM $(0.28 \pm 0.04 \mu \mathrm{g} / \mathrm{ng}$ of dsDNA $)$ aggregates, suggesting that cells did not significantly synthesize de novo collagen in both culture regimens. Additionally, CM+ECM aggregates maintained a significantly higher collagen content than CM aggregates (Figure 2d, upper panel). Collagen content within cell aggregates at day 28 was further evaluated by immunofluorescence, revealing that $\mathrm{CM}+\mathrm{ECM}$ aggregates seem to have a distinct organization of collagen type I fibrils (Figure S2a). PSR staining and polarized light microscopy (PLM) also showed differential collagen fibril organization and composition among the two groups (Figure S2b). Overall, CM + ECM aggregates appear to contain a more substantial amount of collagen type I (yellow) and collagen type III (green), which are essential structural proteins that ensure tensile strength and elasticity in the myocardium.

Although the incorporation of ECM particles did not contribute to a s-GAG enrichment in hiPSC-CM aggregates $(0.77 \pm 0.07 \mu \mathrm{g} / \mathrm{ng}$ of dsDNA and $0.67 \pm 0.12 \mu \mathrm{g} / \mathrm{ng}$ of 


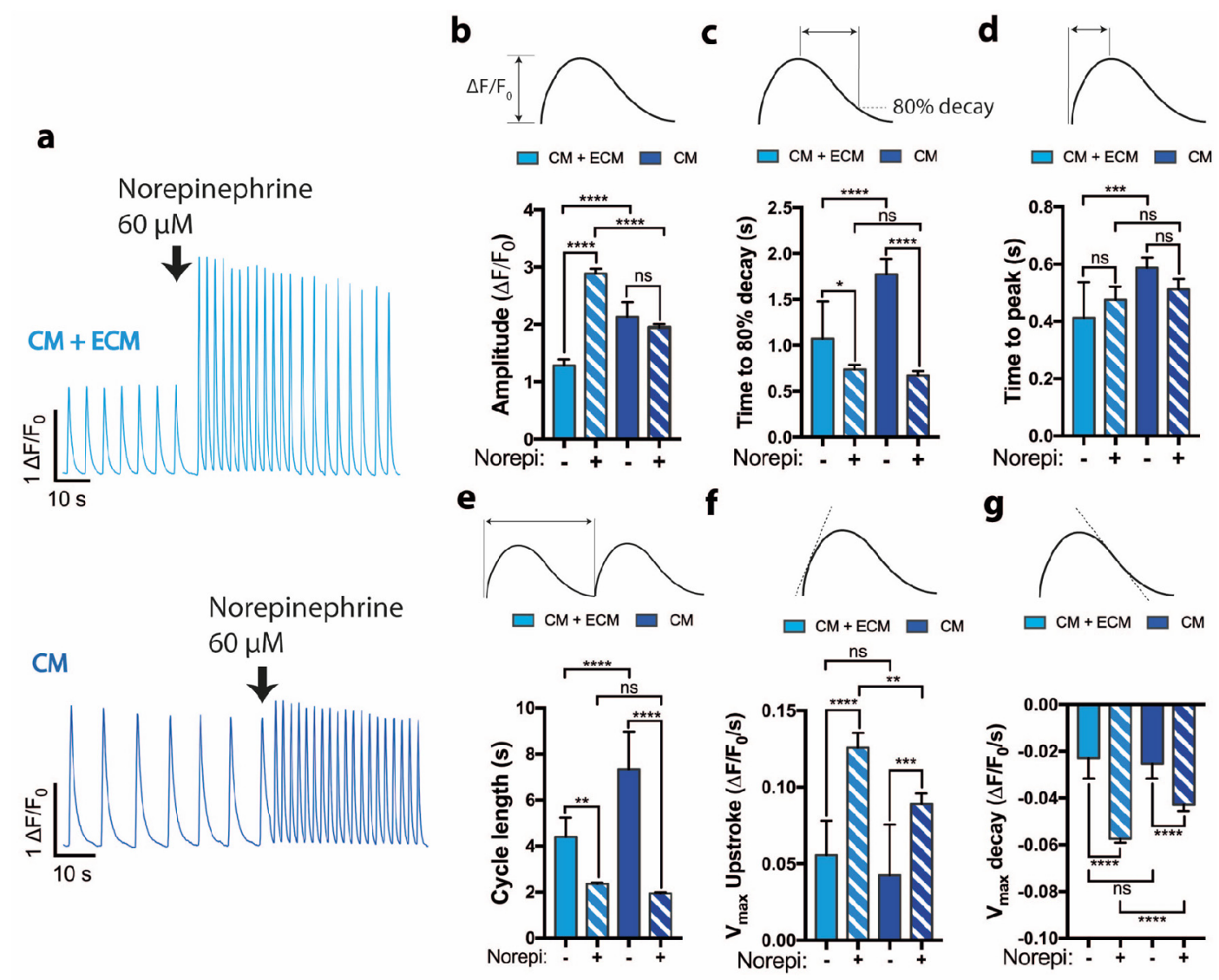

Figure 4. Functional calcium handling of CM+ECM aggregates after 20 days of 3D culture. (a) Representative intracellular calcium transients of $\mathrm{CM}+\mathrm{ECM}$ and $\mathrm{CM}$ aggregates and following administration of $60 \mu \mathrm{M}$ norepinephrine (Norepi). $\Delta F / F_{0}$, change in fluorescence intensity concerning resting fluorescence intensity. ( $\mathrm{b}-\mathrm{g}$ ) CM+ECM (light blue) and CM (dark blue) calcium handling kinetics obtained from the analysis of the transients ( $n=8$ per condition), in the absence (full bars) and presence (stripped bars) of $60 \mu \mathrm{M}$ norepinephrine: (b) amplitude, (c) time to $80 \%$ decay, (d) time to peak, (e) cycle length, (f) upstroke velocity, (g) decay velocity. Throughout, data are presented as mean \pm standard deviation (SD). $* p<0.05, * * p<0.01, * * * p<0.001, * * * p<0.0001$, ns, not significant, determined by one-way ANOVA followed by Tukey's HSD test.

dsDNA in CM+ECM and CM groups, respectively), by day 28, CM+ECM had a significantly higher s-GAG concentration $(0.50 \pm 0.12 \mu \mathrm{g} / \mathrm{ng}$ of dsDNA) than CM aggregates $(0.07 \pm$ $0.01 \mu \mathrm{g} / \mathrm{ng}$ of dsDNA) (Figure 2d, lower panel). By the end of the culture period, CM aggregates had a steep decrease in sGAG content compared to its initial levels, whereas in CM +ECM aggregates, s-GAG retention was substantially ameliorated. ECM particles appear to have improved s-GAG maintenance and may be advantageous in maintaining aggregate matrix integrity.

Our results confirmed that the functionalization with ECM particles influenced neither cell viability, as confirmed by live/ dead staining throughout time (Figure 2e), nor the size of cell aggregates that displayed very similar values of Ferret diameter when compared to the CM group (Figure S3a). In fact, both cell aggregate groups showed a tendency to reduce its size with culture time that could be related to the shear stress experienced in agitated cultures. In addition, CM+ECM aggregates appear to become less spherical along time in culture (d11:0.80 $\pm 0.13 ; \mathrm{d} 28: 0.68 \pm 0.12)$, which is less noticeable in CM aggregates (d11:0.77 $\pm 0.14 ; \mathrm{d} 28: 0.75 \pm$ 0.11 ;) and with statistically significant differences among models by the experimental end point (Figure S3b). CM $+\mathrm{ECM}$ had a higher tendency to self-aggregate (Figure $2 \mathrm{c}$, upper panel), possibly motivated by the presence of ECM particles, that could explain their less spherical appearance.

Synchronous spontaneous contractility was observed nearly in all aggregates (Figure 2f). At day 11, CM+ECM and CM aggregates presented a similar beating rate (respectively, 31.33 \pm 0.58 and $26.67 \pm 4.73$ beats $\mathrm{min}^{-1}$ ), but only CM+ECM showed a moderate increase in beating rate along time in culture $\left(49.00 \pm 2.00\right.$ beats $\left.\mathrm{min}^{-1}\right)$ that was statistically different from the CM group at day 28 (Figure 2f).

Both $\mathrm{CM}+\mathrm{ECM}$ and $\mathrm{CM}$ aggregates demonstrated similar metabolic activity throughout culture (Figure $2 \mathrm{~g}$ ), even though $\mathrm{CM}+\mathrm{ECM}$ aggregates overall displayed a slightly higher capacity to reduce resazurin along time in culture, relatively to $\mathrm{CM}$ aggregates.

3.3. Phenotypic, Structural, and Ultrastructural Features of Functionalized Cardiac Aggregates. By day 28, CM+ECM aggregates expressed cardiac troponin $\mathrm{T}$ (cTnT) and sarcomeric $\alpha$-actinin, with apparent features of cardiomyocyte striation (Figure 3a). The expression for nonmyocyte cellular populations (stained for $\alpha$-smooth muscle actin and vimentin) was also detected by immunofluorescence microscopy (Figure S4). Flow cytometry analysis confirmed that the cell population was dominated by cardiomyocytes, with a SIRP $\alpha / \beta^{+}$cells percentage of $72.27 \pm 11.99 \%$ in $\mathrm{CM}$ $+\mathrm{ECM}$ aggregates and of $69.90 \pm 12.73 \%$ in $\mathrm{CM}$ aggregates 
(Figure 3b), suggesting that ECM functionalization did not compromise the cells' phenotype and composition within the aggregates.

In both cardiac aggregate models, there was a marked change in the expression of genes associated with an adult-like sarcomeric structural organization. $\mathrm{CM}+\mathrm{ECM}$ and $\mathrm{CM}$ aggregates showed an overtime switch in the expression of ventricular sarcomeric fetal gene isoforms (decreased MYH6, MYL7) to adult gene isoforms (increased MYH7, MYL2, TNNI3) (Figure 3c). This change in the fetal gene program is similar to what happens during cardiac muscle development in utero, as to allow force generation with a higher economy of energy consumption. ${ }^{39}$ Additionally, on day 28, expression of MYH7, MYL2, and TNNI3 was higher in CM+ECM aggregates than in control. This fact confirms not only the advantage of a 3D setting to promote hiPSC-CM maturation ${ }^{19}$ but also that incorporation of ECM particles further improves cardiac muscle structural maturity and preferential hiPSC-CM specification toward a ventricular phenotype.

The ultrastructural assessment revealed both cardiac aggregate models displayed sarcomeres composed of organized Z-lines, A-bands, and I-bands, intercalated discs, and gap junctions along electromechanically coupled cardiomyocytes, and abundant mitochondria with distinct cristae preferentially positioned near the contractile apparatus (Figure $3 \mathrm{~d}$ ). The high density of mitochondria observed is consistent with increased energetic workload needed for muscle contraction, possibly as a result of a switch from a glycolytic to a more oxidative metabolism, ${ }^{40}$ as we have previously shown for $3 \mathrm{D}$ hiPSC-CM aggregates. ${ }^{19}$ The contractile machinery was more developed in CM+ECM aggregates versus control, with longer sarcomeres in length (respectively, $1.74 \pm 0.22 \mu \mathrm{m}$ and $1.58 \pm$ $0.19 \mu \mathrm{m}$; Figure $3 \mathrm{e}$ ) and width (respectively, $424.75 \pm 164.39$ $\mathrm{nm}$ and $261.38 \pm 107.92 \mathrm{~nm}$; Figure $3 \mathrm{f}$ ). However, the average sarcomere length of hiPSC-CM in CM+ECM aggregates is still inferior to those found in adult cardiomyocytes ${ }^{41}$ (i.e., $2.2 \mu \mathrm{m}$ ). Also, myofibril alignment was superior in the CM+ECM group compared to CM aggregates, as shown by the significantly lower values estimated for sarcomere angle dispersion (respectively, $5.52 \pm 2.21$ and $10.39 \pm 5.98$; Figure $3 \mathrm{~g}$ ).

3.4. Calcium Handling of Functionalized Cardiac Aggregates. Calcium handling in $\mathrm{CM}+\mathrm{ECM}$ and $\mathrm{CM}$ aggregates was assessed with a fluorescent calcium indicator, enabling the detection of spontaneous calcium transients in each cardiac contraction cycle (Videos S1, S2, S3, and S4). Estimation of calcium kinetics' parameters was done by analyzing calcium transients, both in unstimulated conditions and upon norepinephrine administration (Figure 4a). ECC depends on trans-sarcolemmal calcium influx through L-type $\mathrm{Ca}^{2+}$ channels and subsequent sarcoplasmic reticulum (SR) calcium release. ${ }^{42}$ Unstimulated CM+ECM aggregates, compared to unstimulated CM, exhibited significantly lower transient amplitude (respectively, $1.29 \pm 0.10 \mathrm{~s}$ and $2.13 \pm$ $0.25 \mathrm{~s}$; Figure 4b), relaxation time at $80 \%$ (respectively, $1.08 \pm$ $0.40 \mathrm{~s}$ and $1.78 \pm 0.17 \mathrm{~s}$; Figure $4 \mathrm{c}$ ), time to peak (respectively, $0.41 \pm 0.12 \mathrm{~s}$ and $0.59 \pm 0.04 \mathrm{~s}$; Figure $4 \mathrm{~d}$ ), and cycle length (respectively, $4.40 \pm 0.83 \mathrm{~s}$ and $7.32 \pm 1.65 \mathrm{~s}$; Figure $4 \mathrm{e}$ ). These results indicate that ECM particles may have contributed to a more organized ECC apparatus, allowing a quicker sarcolemma depolarization, with a subsequently faster SR calcium sequestration and sarcolemma repolarization. In the immature hiPSC-CM trans-sarcolemmal influx of calcium is the main driver in ECC due to an underdeveloped SR. ${ }^{43}$
Furthermore, a responsive $\beta$-adrenergic agonist receptor system is both dependent upon L-type $\mathrm{Ca}^{2+}$ channel functionality and SR calcium reserves, ${ }^{9,44}$ and it is a hallmark of mature cardiac muscle. After being challenged with norepinephrine, a $\beta_{1}$-adrenergic agonist, both $\mathrm{CM}+\mathrm{ECM}$ and $\mathrm{CM}$ aggregates showed positive lusitropic and chronotropic responses, with a decreased relaxation time at $80 \%$ (respectively: $0.74 \pm 0.05 \mathrm{~s}$ and $0.68 \pm 0.04 \mathrm{~s}$; Figure $4 \mathrm{c}$ ), and cycle length $(2.36 \pm 0.05 \mathrm{~s}$ and $1.94 \pm 0.05 \mathrm{~s}$, Figure $4 \mathrm{e})$. Also, when stimulated, $\mathrm{CM}+\mathrm{ECM}$ versus $\mathrm{CM}$ aggregates displayed a significantly higher peak amplitude (respectively, $2.89 \pm 0.08 \mathrm{~s}$ and $1.95 \pm 0.06 \mathrm{~s}$; Figure $4 \mathrm{~b}$ ), maximal upstroke velocity (respectively, $0.13 \pm 0.01 \mathrm{~s}^{-1}$ and $0.09 \pm 0.01 \mathrm{~s}^{-1}$; Figure 4f), and maximal decay velocity (respectively, $-0.06 \pm$ $0.002 \mathrm{~s}^{-1}$ and $-0.04 \pm 0.003 \mathrm{~s}^{-1}$; Figure 4g), possibly suggesting a more significant contribution of the SR in ECC, even though we did not evaluate the functionality of this organelle.

\section{DISCUSSION}

One of the goals of cardiac tissue engineering is to generate in vitro heart-mimicking microtissues to study cardiac biology/ development, model disease, and for reliable drug screening. To date, inducing maturation of hiPSC-CM is a challenge in the field, limiting their use in clinical settings. The most advanced strategies reported to date ${ }^{10,12,20}$ can emulate certain cardiomyocyte features that become only evident in the postnatal period. However, obtaining such mature EHT often requires microfabricated platforms or biofabrication knowhow, as well as the need to adapt existing devices or tools to evaluate tissue performance, rendering them poorly scalable for high-throughput purposes. Also, the biomaterials typically used to engineer these tissues do not fully resemble the cardiac ECM. With this in mind, we took our established hiPSCderived cardiac aggregate model $^{19}$ and developed a novel aggregate microtissue that has improved cardiac mimicry by incorporating a minimal quantity of ECM derived from adult human cardiac tissue that had no use for transplantation purposes (i.e., $3 \mathrm{ng}$ ECM/cell). To understand the feasibility of these aggregates' functionalization, we pooled and processed the tissue (in reduced amounts) to ameliorate limitations regarding tissue heterogeneity and donor-to-donor variability. This novel fully humanized cardiac aggregate retained key ECM components throughout a 20-day 3D culture period and showed improved structural maturity. Additionally, our aggregates can be produced in a relatively cost-effective way and are compatible to be scaled in GMP-compliant bioprocesses.

Currently, organ/tissue decellularization is the best option to obtain an ECM-like biomaterial due to the compositional and structural complexity of biological matrices, even though human tissue scarcity and heterogeneity might discourage such an approach. Perfusion-decellularization of human hearts using detergents and an endonuclease combination greatly reduced dsDNA content, but proteomic analysis of the decellularized scaffolds revealed an $89.14 \%$ reduction of the cardiac proteome, with the maintenance of practically only ECM protein families (collagens, laminins, fibrillins, and proteoglycans). ${ }^{31}$ Decellularizing human myocardial tissue with similar chemical/enzymatic methods, that were previously proven successful for animal tissue, is also subjected to patient-topatient variability. ${ }^{45}$ Our method relied purely on physical approaches and was able to reduce tissue cellularity and 
preserve ECM composition in a reproducible and consistent way among donors. Soluble components, such as s-GAG and soluble collagen, which tend not to be fully preserved in detergent/enzymatic-based decellularization protocols, ${ }^{46}$ were retained with our methodology. Further optimizations to our method may be necessary if complete elimination of cells is required. Also, tissue architecture was thoroughly disrupted, making these decellularized (micrometric) scaffolds unsuitable for tissue bioengineering. However, ECM biomaterials are versatile and have other applications that do not require maintaining scaffold architectural integrity, as we have shown here and others elsewhere. For instance, ECM can be broken down, solubilized, and then assembled into nanofibrous hydrogels ${ }^{47}$ that are useful to restore myocardium mechanical integrity after infarction, ${ }^{48}$ or even as a functional element in advanced biofabrication methodologies to create complex biological tissues. ${ }^{49,50}$

ECM was successfully incorporated in a 3D cardiac aggregate model, contributing to a microenvironment richer in ECM components without compromising cell viability and metabolic activity. Noteworthy, we were able to weigh the ECM particles accurately and include them in the forced aggregation step, within the AgreeWells, with a ratio of $3 \mathrm{ng}$ of ECM per cell used. In this process, some of the ECM is lost, but we ensure that we have ECM particles within the aggregates by applying this ECM/cell ratio. Notably, CM $+\mathrm{ECM}$ aggregates retained these ECM components until the end of the culture period. Our aggregates presented a distinct collagen network and were able to better retain soluble components, such as s-GAG, throughout culture. Dynamic culture conditions have been described to promote s-GAG flushing into the medium, resulting in tissue constructs with poor s-GAG content. ${ }^{51,52}$ We hypothesize an initial ECM enrichment within the aggregate might have contributed in improving s-GAG retention by counteracting shear forces that might be prevalent in dynamic cultures. Also, it is known that the interaction between collagens and s-GAG can mediate tissues' integrity and resilience, and when an imbalance between both components happens, it is common to experience tissue dysfunction. ${ }^{27}$ Since CM+ECM aggregates had a superior collagen content throughout culture and these collagens were differentially organized within the aggregate, this improved structural meshwork might have served as anchoring points to s-GAG (and possible other soluble components or signaling molecules), thus overall improving microtissue matrisomal integrity.

Previous studies have identified the ECM as a relevant contributor in cardiomyocyte differentiation ${ }^{29}$ and maturation. ${ }^{30}$ Here, we described that human cardiac ECM plays a role in hiPSC-CM structural maturation. Our CM+ECM aggregates outperformed $\mathrm{CM}$ aggregates in several ways. In particular, CM+ECM aggregates had a higher gene expression of mature ventricular sarcomeric isoforms and longer sarcomeres with a higher degree of alignment. A more organized contractile apparatus ultimately improved calcium handling, with a noticeably quicker ECC and improved response upon a $\beta_{1}$-adrenergic challenge. These differences may suggest an ECM-enriched microenvironment is positively stimulating hiPSC-CM. Even though our cardiac aggregates were not actively being electromechanically stimulated (as it is common for EHT in elastomeric pillars), evidence suggests passive stretching and resistance to construct contraction imposed by the ECM may be sufficient to mechanically stimulate the cells. ${ }^{53}$ Hence, we can state that this particle size was appropriate to allow ECM incorporation within the aggregates, stimulating the cells and promoting maturation, without compromising their 3D structure. We have also observed that our $\mathrm{CM}+\mathrm{ECM}$ aggregates had a moderate increase in beating frequency throughout culture. Automaticity is a hallmark of hiPSC-CM electrophysiological immaturity; ${ }^{54}$ however, early loss of spontaneous contraction may negatively impact structural maturation. In fact, overexpression of KCNJ2 eliminated the phase 4-like depolarization of hESC-CM and ceased their spontaneous firing but led to the downregulation of the contractile apparatus. ${ }^{55}$ Therefore, during maturation, active contraction motivated by an increasing beating frequency may be beneficial to first induce cytoskeleton reorganization, as we verified in our ECM functionalized aggregates, and loss of automaticity later on may improve electrophysiological maturity. As a matter of fact, the fetal heart has a beating frequency superior to the adult heart ${ }^{56}$ (i.e., $2-3$ $\mathrm{Hz}$ versus $1 \mathrm{~Hz}$ ), which has inspired the development of supraphysiological pacing regimens to promote cardiac muscle structural maturation. ${ }^{10-12}$ Even though our CM+ECM aggregates did not develop features that are hallmarks of an adult-like ECC apparatus, such as T-tubulation and a positive FFR, it has been documented that aggregates can effectively be electrically stimulated in microbioreactor systems. ${ }^{57}$ Combining both an ECM-enriched environment, as we have described, and providing electrical stimulation could further improve our aggregate performance and its predictive capacity for precision medicine applications.

Cardiac aggregates present several limitations relative to some EHT models, as they do not allow precise measurement of contractile force and cannot fully recreate the aligned bundle-like appearance of cardiac muscle. Nonetheless, due to their simplicity and high-throughput compatibility, we envision microtissues with improved cardiac mimicry, like the one presented here, could still be powerful tools for drug screening/discovery and disease modeling. In fact, recent studies have reported cardiac microtissues/organoids as reliable platforms in recapitulating heart disease, either by modulating environmental factors in healthy microtissues ${ }^{58}$ or engineering patient-specific microtissues carrying disease susceptibilities. ${ }^{14}$ As ECM dysfunction and remodeling are hallmarks of postmyocardial infarction injury, ${ }^{27}$ it would be relevant to investigate if hiPSC-CM would behave differently if aggregated with pathological human ECM. A recent EHT grown from hiPSC-CM in combination with ECM decellularized from hypertrophic cardiomyopathy porcine tissue biopsies presented abnormal contractile mechanics and calcium handling. ${ }^{59}$ Validating these results in our humanized aggregate model could further validate its use in disease modeling as well as contribute for a better understanding of the ECM's role in heart disease to guide the development of innovative cardiac therapies.

\section{CONCLUSION}

In the current work, we demonstrated that human cardiac myocardial ECM can be decellularized, isolated, fragmented, and, for the first time, successfully incorporated in 3D hiPSC$\mathrm{CM}$ aggregates. The constructs denoted high cell viability and metabolic activity throughout 20 days in agitated culture conditions. The incorporation of the ECM was possible in microtissues by disrupting human cardiac tissue architecture through a physical decellularization approach, even though this 
did not result in substantial loss of its functional components. We demonstrate that human cardiac ECM promotes the phenotypic, structural, and ultrastructural maturation of cardiomyocytes, which further improves calcium handling. We believe that we developed an innovative methodology using cryomilled human ECM to mature hiPSC-CM within $3 \mathrm{D}$ aggregates. This approach has several advantages compared with hydrogels with solubilized ECM, which include the maintenance of the cardiac tissue's micromechanical and compositional properties. Hence, the knowledge generated with this work delivers important insights to streamline the application of more physiologically relevant hiPSC-CM-based tissues to be used in cardiac tissue engineering applications.

\section{ASSOCIATED CONTENT}

\section{SI Supporting Information}

The Supporting Information is available free of charge at https://pubs.acs.org/doi/10.1021/acsabm.0c01490.

$\mathrm{X}$-ray spectroscopy mapping of particles surface; qualitative assessment of collagen network organization and composition of $\mathrm{CM}+\mathrm{ECM}$ and $\mathrm{CM}$ aggregates at day 28; aggregate diameter and sphericity along time in culture; non-myocyte cell population present in $\mathrm{CM}$ $+\mathrm{ECM}$ and CM aggregates at day 28 (PDF)

Spontaneously beating CM+ECM aggregate after 20 days of 3D culture (MOV)

Spontaneously beating CM+ECM aggregate after 20 days of $3 \mathrm{D}$ culture and exposed to $60 \mu \mathrm{M}$ of norepinephrine, resulting in faster contraction (MOV) Spontaneously beating CM aggregate after 20 days of 3D culture (MOV)

Spontaneously beating CM aggregate after 20 days of $3 \mathrm{D}$ culture and exposed to $60 \mu \mathrm{M}$ of norepinephrine, resulting in faster contraction (MOV)

\section{AUTHOR INFORMATION}

\section{Corresponding Author}

Margarida Serra - iBET, Instituto de Biologia Experimental e Tecnológica, 2781-901 Oeiras, Portugal; Instituto de Tecnologia Química e Biológica António Xavier, Universidade

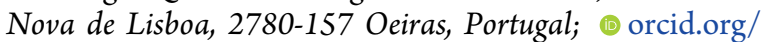
0000-0001-5013-6625; Phone: +351 2144694 31; Email: mserra@ibet.pt

\section{Authors}

Henrique V. Almeida - iBET, Instituto de Biologia Experimental e Tecnológica, 2781-901 Oeiras, Portugal; Instituto de Tecnologia Química e Biológica António Xavier, Universidade Nova de Lisboa, 2780-157 Oeiras, Portugal; (1) orcid.org/0000-0001-9123-4475

Miguel F. Tenreiro - iBET, Instituto de Biologia Experimental e Tecnológica, 2781-901 Oeiras, Portugal; Instituto de Tecnologia Química e Biológica António Xavier, Universidade Nova de Lisboa, 2780-157 Oeiras, Portugal

Ana F. Louro - iBET, Instituto de Biologia Experimental e Tecnológica, 2781-901 Oeiras, Portugal; Instituto de Tecnologia Química e Biológica António Xavier, Universidade Nova de Lisboa, 2780-157 Oeiras, Portugal

Bernardo Abecasis - iBET, Instituto de Biologia Experimental e Tecnológica, 2781-901 Oeiras, Portugal; Instituto de Tecnologia Química e Biológica António Xavier, Universidade Nova de Lisboa, 2780-157 Oeiras, Portugal
Deolinda Santinha - CNC, Centro de Neurociências e Biologia Celular and Faculdade de Medicina, Universidade de Coimbra, 3004-517 Coimbra, Portugal

Tomás Calmeiro - CENIMAT|i3N, Departamento de Ciência dos Materiais, Faculdade de Ciências e Tecnologia, Universidade Nova de Lisboa, 2829-516 Caparica, Portugal

Elvira Fortunato - CENIMAT $\mid i 3 N$, Departamento de Ciência dos Materiais, Faculdade de Ciências e Tecnologia, Universidade Nova de Lisboa, 2829-516 Caparica, Portugal

Lino Ferreira - CNC, Centro de Neurociências e Biologia Celular and Faculdade de Medicina, Universidade de Coimbra, 3004-517 Coimbra, Portugal

Paula M. Alves - iBET, Instituto de Biologia Experimental e Tecnológica, 2781-901 Oeiras, Portugal; Instituto de Tecnologia Química e Biológica António Xavier, Universidade

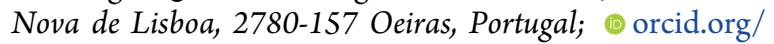
0000-0003-1445-3556

Complete contact information is available at:

https://pubs.acs.org/10.1021/acsabm.0c01490

\section{Author Contributions}

\#HVA and MFT contributed equally to this work. HVA, MFT, and MS conceived the study. HVA, MFT, and AFL designed and executed the experiments. MFT, HVA, and BA performed data analysis and interpretation. DS and LF provided human cardiac tissue. TC and EF performed SEM and AFM analyses. HVA, MFT, and MS wrote the manuscript. All authors revised and approved the manuscript. MS coordinated the study. Funding acquisition was performed by MS, PMA, and LF.

\section{Funding}

The work here presented was funded by Fundação para a Ciência e Tecnologia (FCT) projects NETDIAMOND (SAICTPAC/0047/2015), financially supported by FEEILisboa2020 and FCT/POCI-01-0145-FEDER-016385, and MetaCardio (PTDC/BTM-SAL/32566/2017); iNOVA4Health -UIDB/04462/2020 and UIDP/04462/2020, a program financially supported by FCT/Ministério da Ciência, Tecnologia e Ensino Superior, through national funds is acknowledged; Funding from INTERFACE Programme, through the Innovation, Technology and Circular Economy Fund (FITEC), is gratefully acknowledged; and EU-funded projects BRAV3 (H2020, ID:874827) and ERAatUC (ref. 669088). HVA, AFL, and DS were financed by FCT Grants SFRH/BPD/120595/2016 and PD/BD/139078/2018 and $\mathrm{PD} / \mathrm{BD} / 106051 / 2015$, respectively.

\section{Notes}

The authors declare no competing financial interest.

\section{ACKNOWLEDGMENTS}

The authors acknowledge Joana I. Almeida, from iBET, for the support in flow cytometry and qRT-PCR analyses; Pedro DíazPayno and Daniel Kelly, from Trinity College Dublin, for their support in PLM imaging; A. L. Sousa and E. M. Tranfield from the Electron Microscopy Facility at the Instituto Gulbenkian de Ciência (IGC) for the technical support in TEM; G. Martins from the Advanced Imaging Facility at IGC for the technical support in immunofluorescence microscopy; and the Flow Cytometry and Histopathology units at IGC.

\section{REFERENCES}

(1) Bergmann, O.; Bhardwaj, R. D.; Bernard, S.; Zdunek, S.; Barnabé-Heide, F.; Walsh, S.; Zupicich, J.; Alkass, K.; Buchholz, B. A.; 
Druid, H.; Jovinge, S.; Frisén, J. Evidence for Cardiomyocyte Renewal in Humans. Science (Washington, DC, U. S.) 2009, 324 (5923), 98102.

(2) Banyasz, T.; Lozinskiy, I.; Payne, C. E.; Edelmann, S.; Norton, B.; Chen, B.; Chen-Izu, Y.; Izu, L. T.; Balke, C. W. Transformation of Adult Rat Cardiac Myocytes in Primary Culture. Exp. Physiol. 2008, 93 (3), 370-382.

(3) Fischer, C.; Milting, H.; Fein, E.; Reiser, E.; Lu, K.; Seidel, T.; Schinner, C.; Schwarzmayr, T.; Schramm, R.; Tomasi, R.; Husse, B.; Cao-Ehlker, X.; Pohl, U.; Dendorfer, A. Long-Term Functional and Structural Preservation of Precision-Cut Human Myocardium under Continuous Electromechanical Stimulation in Vitro. Nat. Commun. 2019, 10 (1), 1-12.

(4) Shiba, Y.; Gomibuchi, T.; Seto, T.; Wada, Y.; Ichimura, H.; Tanaka, Y.; Ogasawara, T.; Okada, K.; Shiba, N.; Sakamoto, K.; Ido, D.; Shiina, T.; Ohkura, M.; Nakai, J.; Uno, N.; Kazuki, Y.; Oshimura, M.; Minami, I.; Ikeda, U. Allogeneic Transplantation of IPS CellDerived Cardiomyocytes Regenerates Primate Hearts. Nature 2016, 538, 388-391.

(5) Burridge, P. W.; Li, Y. F.; Matsa, E.; Wu, H.; Ong, S. G.; Sharma, A.; Holmström, A.; Chang, A. C.; Coronado, M. J.; Ebert, A. D.; Knowles, J. W.; Telli, M. L.; Witteles, R. M.; Blau, H. M.; Bernstein, D.; Altman, R. B.; Wu, J. C. Human Induced Pluripotent Stem CellDerived Cardiomyocytes Recapitulate the Predilection of Breast Cancer Patients to Doxorubicin-Induced Cardiotoxicity. Nat. Med. 2016, 22 (5), 547-556.

(6) Yazawa, M.; Hsueh, B.; Jia, X.; Pasca, A. M.; Bernstein, J. A.; Hallmayer, J.; Dolmetsch, R. E. Using Induced Pluripotent Stem Cells to Investigate Cardiac Phenotypes in Timothy Syndrome. Nature 2011, 471 (7337), 230-236.

(7) Burridge, P. W.; Matsa, E.; Shukla, P.; Lin, Z. C.; Churko, J. M.; Ebert, A. D.; Lan, F.; Diecke, S.; Huber, B.; Mordwinkin, N. M.; Plews, J. R.; Abilez, O. J.; Cui, B.; Gold, J. D.; Wu, J. C. Chemically Defned Generation of Human Cardiomyocytes. Nat. Methods 2014, 11 (8), 855-860.

(8) Veerman, C. C.; Kosmidis, G.; Mummery, C. L.; Casini, S.; Verkerk, A. O.; Bellin, M. Immaturity of Human Stem-Cell-Derived Cardiomyocytes in Culture: Fatal Flaw or Soluble Problem? Stem Cells Dev. 2015, 24, 1035.

(9) Mannhardt, I.; Breckwoldt, K.; Letuffe-Brenière, D.; Schaaf, S.; Schulz, H.; Neuber, C.; Benzin, A.; Werner, T.; Eder, A.; Schulze, T.; Klampe, B.; Christ, T.; Hirt, M. N.; Huebner, N.; Moretti, A.; Eschenhagen, T.; Hansen, A. Human Engineered Heart Tissue: Analysis of Contractile Force. Stem Cell Rep. 2016, 7 (1), 29-42.

(10) Ronaldson-Bouchard, K.; Ma, S. P.; Yeager, K.; Chen, T.; Song, L. J.; Sirabella, D.; Morikawa, K.; Teles, D.; Yazawa, M.; VunjakNovakovic, G. Advanced Maturation of Human Cardiac Tissue Grown from Pluripotent Stem Cells. Nature 2018, 556, 239.

(11) Nunes, S. S.; Miklas, J. W.; Liu, J.; Aschar-Sobbi, R.; Xiao, Y.; Zhang, B.; Jiang, J.; Massé, S.; Gagliardi, M.; Hsieh, A.; Thavandiran, N.; Laflamme, M. A.; Nanthakumar, K.; Gross, G. J.; Backx, P. H.; Keller, G.; Radisic, M. Biowire: A Platform for Maturation of Human Pluripotent Stem Cell-Derived Cardiomyocytes. Nat. Methods 2013, $10(8), 781-787$.

(12) Zhao, Y.; Rafatian, N.; Feric, N. T.; Cox, B. J.; Aschar-Sobbi, R.; Wang, E. Y.; Aggarwal, P.; Zhang, B.; Conant, G.; RonaldsonBouchard, K.; Pahnke, A.; Protze, S.; Lee, J. H.; Davenport Huyer, L.; Jekic, D.; Wickeler, A.; Naguib, H. E.; Keller, G. M.; VunjakNovakovic, G.; Broeckel, U.; Backx, P. H.; Radisic, M. A Platform for Generation of Chamber-Specific Cardiac Tissues and Disease Modeling. Cell 2019, 176 (4), 913-927.

(13) Abecasis, B.; Gomes-Alves, P.; Rosa, S.; Gouveia, P. J.; Ferreira, L.; Serra, M.; Alves, P. M. Unveiling the Molecular Crosstalk in a Human Induced Pluripotent Stem Cell-Derived Cardiac Model. Biotechnol. Bioeng. 2019, 116, 1245.

(14) Giacomelli, E.; Meraviglia, V.; Campostrini, G.; Cochrane, A.; Cao, X.; van Helden, R. W. J.; Krotenberg Garcia, A.; Mircea, M.; Kostidis, S.; Davis, R. P.; van Meer, B. J.; Jost, C. R.; Koster, A. J.; Mei, H.; Míguez, D. G.; Mulder, A. A.; Ledesma-Terrón, M.;
Pompilio, G.; Sala, L.; Salvatori, D. C. F.; Slieker, R. C.; Sommariva, E.; de Vries, A. A. F.; Giera, M.; Semrau, S.; Tertoolen, L. G. J.; Orlova, V. V.; Bellin, M.; Mummery, C. L. Human-IPSC-Derived Cardiac Stromal Cells Enhance Maturation in 3D Cardiac Microtissues and Reveal Non-Cardiomyocyte Contributions to Heart Disease. Cell Stem Cell 2020, 26, 862.

(15) Correia, C.; Koshkin, A.; Duarte, P.; Hu, D.; Teixeira, A.; Domian, I.; Serra, M.; Alves, P. M. Distinct Carbon Sources Affect Structural and Functional Maturation of Cardiomyocytes Derived from Human Pluripotent Stem Cells. Sci. Rep. 2017, 7 (1), 8590.

(16) Hu, D.; Linders, A.; Yamak, A.; Correia, C.; Kijlstra, J. D.; Garakani, A.; Xiao, L.; Milan, D. J.; van der Meer, P.; Serra, M.; Alves, P. M.; Domian, I. J. Metabolic Maturation of Human Pluripotent Stem Cell-Derived Cardiomyocytes by Inhibition of HIF $1 \alpha$ and LDHA. Circ. Res. 2018, 123 (9), 1066-1079.

(17) Yang, X.; Rodriguez, M.; Pabon, L.; Fischer, K. A.; Reinecke, H.; Regnier, M.; Sniadecki, N. J.; Ruohola-Baker, H.; Murry, C. E. Tri-Iodo-l-Thyronine Promotes the Maturation of Human Cardiomyocytes-Derived from Induced Pluripotent Stem Cells. J. Mol. Cell. Cardiol. 2014, 72, 296.

(18) Parikh, S. S.; Blackwell, D. J.; Gomez-Hurtado, N.; Frisk, M.; Wang, L.; Kim, K.; Dahl, C. P.; Fiane, A.; Tønnessen, T.; Kryshtal, D. O.; Louch, W. E.; Knollmann, B. C. Thyroid and Glucocorticoid Hormones Promote Functional T-Tubule Development in HumanInduced Pluripotent Stem Cell-Derived Cardiomyocytes. Circ. Res. 2017, 121 (12), 1323-1330.

(19) Correia, C.; Koshkin, A.; Duarte, P.; Hu, D.; Carido, M.; Sebastião, M. J.; Gomes-Alves, P.; Elliott, D. A.; Domian, I. J.; Teixeira, A. P.; Alves, P. M.; Serra, M. 3D Aggregate Culture Improves Metabolic Maturation of Human Pluripotent Stem Cell Derived Cardiomyocytes. Biotechnol. Bioeng. 2018, 115 (3), 630-644.

(20) Shadrin, I. Y.; Allen, B. W.; Qian, Y.; Jackman, C. P.; Carlson, A. L.; Juhas, M. E.; Bursac, N. Cardiopatch Platform Enables Maturation and Scale-up of Human Pluripotent Stem Cell-Derived Engineered Heart Tissues. Nat. Commun. 2017, 8, 1825.

(21) MacQueen, L. A.; Sheehy, S. P.; Chantre, C. O.; Zimmerman, J. F.; Pasqualini, F. S.; Liu, X.; Goss, J. A.; Campbell, P. H.; Gonzalez, G. M.; Park, S.-J.; Capulli, A. K.; Ferrier, J. P.; Kosar, T. F.; Mahadevan, L.; Pu, W. T.; Parker, K. K. A Tissue-Engineered Scale Model of the Heart Ventricle. Nat. Biomed. Eng. 2018, 2 (12), 930-941.

(22) Kobayashi, J.; Okano, T. Design of Temperature-Responsive Polymer-Grafted Surfaces for Cell Sheet Preparation and Manipulation. Bull. Chem. Soc. Jpn. 2019, 92, 817.

(23) Spicer, C. D. Hydrogel Scaffolds for Tissue Engineering: The Importance of Polymer Choice. Polym. Chem. 2020, 11, 184.

(24) Song, J.; Jia, X.; Minami, K.; Hill, J. P.; Nakanishi, J.; Shrestha, L. K.; Ariga, K. Large-Area Aligned Fullerene Nanocrystal Scaffolds as Culture Substrates for Enhancing Mesenchymal Stem Cell SelfRenewal and Multipotency. ACS Appl. Nano Mater. 2020, 3, 6497.

(25) Schwach, V.; Passier, R. Native Cardiac Environment and Its Impact on Engineering Cardiac Tissue. Biomater. Sci. 2019, 7 (9), 3566-3580.

(26) Bassat, E.; Mutlak, Y. E.; Genzelinakh, A.; Shadrin, I. Y.; Baruch Umansky, K.; Yifa, O.; Kain, D.; Rajchman, D.; Leach, J.; Riabov Bassat, D.; Udi, Y.; Sarig, R.; Sagi, I.; Martin, J. F.; Bursac, N.; Cohen, S.; Tzahor, E. The Extracellular Matrix Protein Agrin Promotes Heart Regeneration in Mice. Nature 2017, 547 (7662), 179-184.

(27) Rienks, M.; Papageorgiou, A. P.; Frangogiannis, N. G.; Heymans, S. Myocardial Extracellular Matrix: An Ever-Changing and Diverse Entity. Circ. Res. 2014, 114, 872.

(28) Chanthra, N.; Abe, T.; Miyamoto, M.; Sekiguchi, K.; Kwon, C.; Hanazono, Y.; Uosaki, H. A Novel Fluorescent Reporter System Identifies Laminin-511/521 as Potent Regulators of Cardiomyocyte Maturation. Sci. Rep. 2020, 10, 4249 DOI: 10.1038/s41598-02061163-3.

(29) Higuchi, S.; Lin, Q.; Wang, J.; Lim, T. K.; Joshi, S. B.; Anand, G. S.; Chung, M. C. M.; Sheetz, M. P.; Fujita, H. Heart Extracellular Matrix Supports Cardiomyocyte Differentiation of Mouse Embryonic Stem Cells. J. Biosci. Bioeng. 2013, 115 (3), 320-325. 
(30) Fong, A. H.; Romero-López, M.; Heylman, C. M.; Keating, M.; Tran, D.; Sobrino, A.; Tran, A. Q.; Pham, H. H.; Fimbres, C.; Gershon, P. D.; Botvinick, E. L.; George, S. C.; Hughes, C. C. W. Three-Dimensional Adult Cardiac Extracellular Matrix Promotes Maturation of Human Induced Pluripotent Stem Cell-Derived Cardiomyocytes. Tissue Eng., Part A 2016, 22 (15-16), 1016-1025.

(31) Guyette, J. P.; Charest, J. M.; Mills, R. W.; Jank, B. J.; Moser, P. T.; Gilpin, S. E.; Gershlak, J. R.; Okamoto, T.; Gonzalez, G.; Milan, D. J.; Gaudette, G. R.; Ott, H. C. Bioengineering Human Myocardium on Native Extracellular Matrix. Circ. Res. 2016, 118 (1), 56-72.

(32) Correia, C.; Koshkin, A.; Carido, M.; Espinha, N.; Šarić, T.; Lima, P. A.; Serra, M.; Alves, P. M. Effective Hypothermic Storage of Human Pluripotent Stem Cell-Derived Cardiomyocytes Compatible With Global Distribution of Cells for Clinical Applications and Toxicology Testing. Stem Cells Transl. Med. 2016, 5 (5), 658-669.

(33) Serra, M.; Correia, C.; Malpique, R.; Brito, C.; Jensen, J.; Bjorquist, P.; Carrondo, M. J. T.; Alves, P. M. Microencapsulation Technology: A Powerful Tool for Integrating Expansion and Cryopreservation of Human Embryonic Stem Cells. PLoS One 2011, 6, e23212.

(34) Abecasis, B.; Canhão, P. G. M.; Almeida, H. V.; Calmeiro, T.; Fortunato, E.; Gomes-Alves, P.; Serra, M.; Alves, P. M. Toward a Microencapsulated 3D HiPSC-Derived in Vitro Cardiac Microtissue for Recapitulation of Human Heart Microenvironment Features. Front. Bioeng. Biotechnol. 2020, 8, 1163.

(35) Cortiella, J.; Niles, J.; Cantu, A.; Brettler, A.; Pham, A.; Vargas, G.; Winston, S.; Wang, J.; Walls, S.; Nichols, J. E. Influence of Acellular Natural Lung Matrix on Murine Embryonic Stem Cell Differentiation and Tissue Formation. Tissue Eng., Part A 2010, 16, 2565.

(36) Hopkinson, A.; Shanmuganathan, V. A.; Gray, T.; Yeung, A. M.; Lowe, J.; James, D. K.; Dua, H. S. Optimization of Amniotic Membrane (AM) Denuding for Tissue Engineering. Tissue Eng., Part C 2008, 14, 371.

(37) Funamoto, S.; Nam, K.; Kimura, T.; Murakoshi, A.; Hashimoto, Y.; Niwaya, K.; Kitamura, S.; Fujisato, T.; Kishida, A. The Use of High-Hydrostatic Pressure Treatment to Decellularize Blood Vessels. Biomaterials 2010, 31, 3590.

(38) Sutherland, A. J.; Beck, E. C.; Dennis, S. C.; Converse, G. L.; Hopkins, R. A.; Berkland, C. J.; Detamore, M. S. Decellularized Cartilage May Be a Chondroinductive Material for Osteochondral Tissue Engineering. PLoS One 2015, 10, e0121966.

(39) Reiser, P. J.; Portman, M. A.; Ning, X. H.; Moravec, C. S. Human Cardiac Myosin Heavy Chain Isoforms in Fetal and Failing Adult Atria and Ventricles. Am. J. Physiol. - Hear. Circ. Physiol. 2001, 280, H1814.

(40) Porter, G. A.; Hom, J. R.; Hoffman, D. L.; Quintanilla, R. A.; Bentley, K. L. d. M.; Sheu, S. S. Bioenergetics, Mitochondria, and Cardiac Myocyte Differentiation. Prog. Pediatr. Cardiol. 2011, 31 (2), $75-81$.

(41) Gerdes, A. M.; Kellerman, S. E.; Moore, J. A.; Muffly, K. E.; Clark, L. C.; Reaves, P. Y.; Malec, K. B.; McKeown, P. P.; Schocken, D. D. Structural Remodeling of Cardiac Myocytes in Patients with Ischemic Cardiomyopathy. Circulation 1992, 86 (2), 426-430.

(42) Bers, D. M. Cardiac Excitation-Contraction Coupling. Nature 2002, 415 (6868), 198-205.

(43) Liu, J.; Fu, J. D.; Siu, C. W.; Li, R. A. Functional Sarcoplasmic Reticulum for Calcium Handling of Human Embryonic Stem CellDerived Cardiomyocytes: Insights for Driven Maturation. Stem Cells 2007, 25 (12), 3038-3044.

(44) Huebsch, N.; Loskill, P.; Deveshwar, N.; Spencer, C. I.; Judge, L. M.; Mandegar, M. A.; Fox, C. B.; Mohamed, T. M. A.; Ma, Z.; Mathur, A.; Sheehan, A. M.; Truong, A.; Saxton, M.; Yoo, J.; Srivastava, D.; Desai, T. A.; So, P. L.; Healy, K. E.; Conklin, B. R. Miniaturized IPS-Cell-Derived Cardiac Muscles for Physiologically Relevant Drug Response Analyses. Sci. Rep. 2016, 6, 1-12.

(45) Johnson, T. D.; Dequach, J. A.; Gaetani, R.; Ungerleider, J.; Elhag, D.; Nigam, V.; Behfar, A.; Christman, K. L. Human versus
Porcine Tissue Sourcing for an Injectable Myocardial Matrix Hydrogel. Biomater. Sci. 2014, 2 (5), 735-744.

(46) Partington, L.; Mordan, N. J.; Mason, C.; Knowles, J. C.; Kim, H. W.; Lowdell, M. W.; Birchall, M. A.; Wall, I. B. Biochemical Changes Caused by Decellularization May Compromise Mechanical Integrity of Tracheal Scaffolds. Acta Biomater. 2013, 9, 5251-5261.

(47) Seif-Naraghi, S. B.; Salvatore, M. A.; Schup-Magoffin, P. J.; Hu, D. P.; Christman, K. L. Design and Characterization of an Injectable Pericardial Matrix Gel: A Potentially Autologous Scaffold for Cardiac Tissue Engineering. Tissue Eng., Part A 2010, 16 (6), 2017-2027.

(48) Seif-Naraghi, S. B.; Singelyn, J. M.; Salvatore, M. A.; Osborn, K. G.; Wang, J. J.; Sampat, U.; Kwan, O. L.; Strachan, G. M.; Wong, J.; Schup-Magoffin, P. J.; Braden, R. L.; Bartels, K.; DeQuach, J. A.; Preul, M.; Kinsey, A. M.; DeMaria, A. N.; Dib, N.; Christman, K. L. Safety and Efficacy of an Injectable Extracellular Matrix Hydrogel for Treating Myocardial Infarction. Sci. Transl. Med. 2013, 5 (173), 173 ra25.

(49) Pati, F.; Jang, J.; Ha, D. H.; Won Kim, S.; Rhie, J. W.; Shim, J. H.; Kim, D. H.; Cho, D. W. Printing Three-Dimensional Tissue Analogues with Decellularized Extracellular Matrix Bioink. Nat. Commun. 2014, 5, 3935 DOI: 10.1038/ncomms4935.

(50) Noor, N.; Shapira, A.; Edri, R.; Gal, I.; Wertheim, L.; Dvir, T. 3D Printing of Personalized Thick and Perfusable Cardiac Patches and Hearts. Adv. Sci. 2019, 6, 1900344.

(51) Shahin, K.; Doran, P. M. Strategies for Enhancing the Accumulation and Retention of Extracellular Matrix in TissueEngineered Cartilage Cultured in Bioreactors. PLoS One 2011, 6, e23119.

(52) Freyria, A. M.; Yang, Y.; Chajra, H.; Rousseau, C. F.; Ronzière, M. C.; Herbage, D.; El Haj, A. J. Optimization of Dynamic Culture Conditions: Effects on Biosynthetic Activities of Chondrocytes Grown in Collagen Sponges. Tissue Eng. 2005, 11 (5-6), 674-684.

(53) Gonen-Wadmany, M.; Gepstein, L.; Seliktar, D. Controlling the Cellular Organization of Tissue-engineered Cardiac Constructs. Ann. N. Y. Acad. Sci. 2004, 1015 (1), 299-311.

(54) Satin, J.; Kehat, I.; Caspi, O.; Huber, I.; Arbel, G.; Itzhaki, I.; Magyar, J.; Schroder, E. A.; Perlman, I.; Gepstein, L. Mechanism of Spontaneous Excitability in Human Embryonic Stem Cell Derived Cardiomyocytes. J. Physiol. 2004, 559 (2), 479-496.

(55) Lieu, D. K.; Fu, J. D.; Chiamvimonvat, N.; Tung, K. C.; McNerney, G. P.; Huser, T.; Keller, G.; Kong, C. W.; Li, R. A. Mechanism-Based Facilitated Maturation of Human Pluripotent Stem Cell-Derived Cardiomyocytes. Circ.: Arrhythmia Electrophysiol. 2013, 6 (1), 191-201.

(56) Shuffrey, L. C.; Myers, M. M.; Odendaal, H. J.; Elliott, A. J.; du Plessis, C.; Groenewald, C.; Burd, L.; Angal, J.; Nugent, J. D.; Isler, J. R.; Fifer, W. P. Fetal Heart Rate, Heart Rate Variability, and Heart Rate/Movement Coupling in the Safe Passage Study. J. Perinatol. 2019, 39 (5), 608-618.

(57) Eng, G.; Lee, B. W.; Protas, L.; Gagliardi, M.; Brown, K.; Kass, R. S.; Keller, G.; Robinson, R. B.; Vunjak-Novakovic, G. Autonomous Beating Rate Adaptation in Human Stem Cell-Derived Cardiomyocytes. Nat. Commun. 2016, 7, 1-10.

(58) Richards, D. J.; Li, Y.; Kerr, C. M.; Yao, J.; Beeson, G. C.; Coyle, R. C.; Chen, X.; Jia, J.; Damon, B.; Wilson, R.; Starr Hazard, E.; Hardiman, G.; Menick, D. R.; Beeson, C. C.; Yao, H.; Ye, T.; Mei, Y. Human Cardiac Organoids for the Modelling of Myocardial Infarction and Drug Cardiotoxicity. Nat. Biomed. Eng. 2020, 4 (4), $446-462$.

(59) Sewanan, L. R.; Schwan, J.; Kluger, J.; Park, J.; Jacoby, D. L.; Qyang, Y.; Campbell, S. G. Extracellular Matrix From Hypertrophic Myocardium Provokes Impaired Twitch Dynamics in Healthy Cardiomyocytes. JACC Basic to Transl. Sci. 2019, 4 (4), 495-505. 